\title{
Uso de aparelho de amplificação sonora individual por idosos: estudo SABE - saúde, bem- estar e envelhecimento
}

\author{
Hearing aids use among elderly: SABE study - health, well-being \\ and aging survey
}

\author{
Mariana Sodário Cruz', Maria Cristina Pereira Lima², Jair Lício Ferreira Santos³, Yeda Aparecida de Oliveira \\ Duarte ${ }^{4}$, Maria Lucia Lebrão ${ }^{5}$, Ana Teresa de Abreu Ramos-Cerqueira ${ }^{2}$
}

\begin{abstract}
RESUMO
Objetivo: Descrever o uso do aparelho de amplificação sonora individual (AASI) pela população idosa do município de São Paulo e fatores associados. Métodos: Estudo transversal, descritivo e quantitativo, integrante do Estudo SABE (Saúde, Bem-estar e Envelhecimento), desenvolvido no ano de 2006. Foram entrevistados 1115 sujeitos com 65 anos ou mais. A amostra foi obtida de setores censitários em dois estágios, com reposição e probabilidade proporcional à população, complementando-se para acima de 75 anos. Utilizou-se questionário estruturado e instrumentos validados. Os dados foram ponderados e a análise foi realizada no software Stata $10 \circledR$, através do teste de Rao-Scott para análise univariada e regressão logística tipo stepwise backward para análise multivariada. Resultados: Trezentos e setenta e sete sujeitos $(30,4 \%)$ apresentaram deficiência auditiva autorreferida e destes, $10,1 \%$ declararam utilizar o AASI. Dentre as formas de aquisição do dispositivo, 78,8\% utilizaram recursos particulares e 16,9\% adquiriram via Sistema Único de Saúde (SUS). Dentre os não protetizados, 16,6\% relataram indicação prévia. Entretanto, 8,6\% não se acostumaram e $8,0 \%$ não adquiriram, por problemas financeiros. O uso do AASI associou-se à menor prevalência de provável demência. Conclusão: O baixo número de usuários de AASI indica dificuldades dos idosos em adquiri-lo e/ou dos serviços de saúde em adaptá-los de forma efetiva. Esse achado pode influenciar a qualidade de vida do idoso deficiente auditivo, visto a associação com a provável demência, revelada pelo presente estudo.
\end{abstract}

Descritores: Envelhecimento; Demência; Auxiliares de audição; Perda auditiva; Inquéritos de morbidade

\begin{abstract}
Purpose: Describe hearing aid use by the elderly population in the city of São Paulo and identify associated factors. Methods: A cross-sectional, descriptive, quantitative study integrated with the SABE (health, wellbeing and aging) project developed in 2006. A total of 1.115 individuals aged 65 or over were interviewed. Sample selection occurred in two stages, with replacement and probabilities proportional to the population to complement those aged 75 or over. Structured questionnaires and validated instruments were used. The data were weighted, the Rao-Scott test was used for univariate analysis and backward stepwise logistic regression was used for multivariate analysis, performed on Stata $10^{\circledR}$ software. Results: Three hundred and seventy-seven subjects (30.4\%) were classified as hearing impaired and $10.1 \%$ of these reported using hearing aids. To acquire the devices, $78.8 \%$ used their own resources and $16.9 \%$ acquired them through the Brazilian public health system (SUS). Among non-users of hearing aids, $16.6 \%$ reported prior indication; however, $8.6 \%$ were unable to adapt to the device and $8.0 \%$ could not afford to acquire one. Hearing aid use was associated with lower prevalence of probable dementia. Conclusion: The low number of hearing aid users indicates the difficulties elderly people face in acquiring them and/or that the health services face in effectively helping them to adapt. These findings may influence the quality of life of elderly with hearing impairment, given the association with probable dementia revealed by this study.
\end{abstract}

Keywords: Aging; Dementia; Hearing aids; Hearing loss; Morbidity surveys

Trabalho realizado no Curso de Pós-Graduação em Saúde Coletiva (Doutorado), área de concentração em Saúde Pública, Faculdade de Medicina de Botucatu, Universidade Estadual Paulista "Júlio de Mesquita Filho" - UNESP - Botucatu (SP), Brasil.

(1) Colegiado de Saúde Coletiva da Faculdade de Ceilândia, Universidade de Brasília - UnB - Ceilândia (DF), Brasil.

(2) Departamento de Neurologia, Psicologia e Psiquiatria, Faculdade de Medicina de Botucatu, Universidade Estadual Paulista "Júlio de Mesquita Filho" UNESP - Botucatu (SP), Brasil.

(3) Departamento de Medicina Social, Faculdade de Medicina de Ribeirão Preto, Universidade de São Paulo - USP - Ribeirão Preto (SP), Brasil.

(4) Departamento de Enfermagem Médico-Cirúrgica, Escola de Enfermagem, Universidade de São Paulo - USP - São Paulo (SP), Brasil.

(5) Departamento de Epidemiologia, Faculdade de Saúde Pública, Universidade de São Paulo - USP - São Paulo (SP), Brasil.

Conflitos de interesses: Não

Contribuição dos autores: $M S C$ contribuiu no levantamento bibliográfico, tratamento e análise dos dados, elaboração do manuscrito e revisão final. $M C P L$ contribuiu na concepção e planejamento, análise e interpretação dos dados, elaboração do rascunho e aprovação da versão final do manuscrito. JLFS colaborou no tratamento dos dados, análise estatística e revisão da versão final. YAOD contribuiu para o desenho do estudo, organização do banco de dados, análise e interpretação dos dados e revisão crítica da versão final. $M L L$ colaborou no desenho do estudo e revisão da versão final. ATARC contribuiu na construção do banco e análise dos dados, participou da redação dos resultados e discussão e da revisão final do manuscrito.

Endereço para correspondência: Mariana Sodário Cruz. Conjunto A, Lote 01, Centro Metropolitano, Ceilândia (DF), Brasil, CEP: $72220-900$.

E-mail: msodario@unb.br

Recebido em: 14/8/2012; Aceito em: 15/7/2013 


\section{INTRODUÇÃO}

O envelhecimento da população brasileira exige projetos e pesquisas específicos para a fatia demográfica de idosos, visto que esse grupo etário apresenta características peculiares de saúde física e mental. Dentre as alterações mais comumente encontradas nos idosos, destacam-se os prejuízos sensoriais, tais como a deficiência visual e auditiva, que podem influenciar o comportamento e a interação social.

Presbiacusia é o termo geral utilizado para referir-se à perda auditiva relacionada à idade. Caracteriza-se pela redução da sensibilidade auditiva e da compreensão da fala em ambientes ruidosos, lentidão do processamento central da informação acústica e localização deficiente de fontes sonoras ${ }^{(1)}$. Popularmente, acredita-se que a presbiacusia acomete somente os idosos. Entretanto, vale ressaltar que pode ser detectada em adultos mais jovens, a partir dos 50 anos, ou até mesmo, a partir dos $40 \operatorname{anos}^{(2)}$, sendo sua associação mais importante com o processo de envelhecimento e não com a idade cronológica exclusiva e pontualmente.

Devido à estreita relação entre a perda auditiva e o avanço da idade, pressupõe-se que, com o aumento de idosos na população brasileira, também ocorra o aumento de pessoas com deficiência auditiva (DA), principalmente se ações de saúde coletiva direcionadas aos adultos jovens e idosos não investirem, com a devida importância, em medidas para a promoção da saúde auditiva e prevenção dessa deficiência.

Um dos impactos do crescimento do número de adultos e idosos deficientes auditivos pode ser refletido nos serviços de saúde especializados em saúde auditiva, principalmente os de média e alta complexidade, responsáveis pela adaptação de aparelhos auditivos, segundo a Portaria 2073/04, que estabeleceu a Política Nacional de Atenção à Saúde Auditiva (PNSA). Esse documento indicou as diretrizes do atendimento em saúde auditiva, enfatizando a interdisciplinaridade, a educação continuada de profissionais e as medidas preventivas em todos os níveis. Além disso, estabeleceu diretrizes para a organização dos serviços nas instituições de saúde, em todos os níveis de densidade tecnológica: atenção básica, média e alta complexidade ${ }^{(3)}$.

Apesar da maior organização preconizada pela PNSA, observa-se que a existência de serviços disponíveis não garante que a população os utilize. $\mathrm{O}$ acesso aos serviços de saúde pode relacionar-se a características da oferta. Entretanto, o efetivo uso de serviços, apesar de ser uma expressão do acesso, também depende de fatores individuais e contextuais ${ }^{(4)}$.

A presença de serviços que contemplem ações com enfoque na saúde auditiva pode ser um fator de proteção para a deficiência auditiva, devido ao caráter de prevenção primária e de promoção da saúde que alguns pontos de atenção podem apresentar, tais como as Unidades Básicas de Saúde e as Unidades de Saúde da Família. Apesar da formação das equipes mínimas da estratégia de saúde da família não contar com a participação de profissionais com formação em saúde auditiva, tais como fonoaudiólogos e médicos otorrinolaringologistas, esses profissionais podem compor as equipes do NASF (Núcleo de Apoio à Saúde da Família) e auxiliar nas medidas coletivas de melhoria da saúde auditiva da população, em parceria com os profissionais que compõem as equipes integrantes da estratégia, principalmente após a instituição da Portaria 154 de $24 / 01 / 2008^{(5)}$.

Além da disponibilidade dos serviços e regulamentação da lei, a proximidade geográfica pode ser outro facilitador para o acesso à reabilitação auditiva. Em um estudo qualitativo realizado em serviço de média complexidade em saúde auditiva, localizado em Itajaí (SC), com 19 idosos usuários de aparelho da amplificação sonora individual (AASI), os autores concluíram que os idosos deficientes auditivos atribuíram a possibilidade de acesso ao AASI à implantação do Serviço de Atenção à Saúde Auditiva próximo às regiões de suas moradias, visto que esse tipo serviço tende a organizar a oferta e a disponibilidade dos atendimentos ${ }^{(6)}$.

Apesar dos avanços, o uso efetivo de AASI pela população idosa ainda é um problema de difícil solução para os audiologistas, realidade também apontada em estudos internacionais. Em pesquisa realizada na Finlândia, constatou-se que apenas 15 a 30\% de idosos com deficiência auditiva possuíam AASI. Desses, somente 55\% faziam uso do dispositivo em tempo integral. As principais razões para a não utilização do AASI foram: não percepção de melhora, quebra do aparelho e, por último, a dificuldade de manuseio do equipamento, em função do tamanho reduzido das próteses auditivas, especialmente considerando-se a presença frequente de alterações motoras finas em alguns idosos ${ }^{(7)}$. Esses dados são preocupantes, tendo em vista os possíveis benefícios que o uso do AASI pode oferecer, tais como melhoria nas situações de comunicação e contribuição para a preservação da capacidade funcional e das habilidades cognitivas ${ }^{(8)}$.

O Brasil ainda é carente de estudos que relacionem a prevalência da deficiência auditiva (DA) com a presença de serviços de saúde, ou que descrevam o acesso da população com DA a esses serviços. Obter informações sobre o uso dos serviços pelos idosos com DA pode auxiliar na melhor formulação de políticas públicas que visem à saúde auditiva e a potencializar as ações dos serviços já existentes.

Para atender a essa e a outras demandas de informação na área da saúde, especialmente voltadas para os idosos, teve início, no ano de 2000, o Estudo SABE - Saúde, Bem-estar e Envelhecimento. Inicialmente, o estudo foi proposto e coordenado pela Organização Pan-Americana de Saúde, como uma proposta multicêntrica com a participação da Argentina, Barbados, Brasil, Chile, Cuba, México e Uruguai. Caracterizouse por ser simultâneo, abrangente e rigorosamente comparável, sendo o primeiro desse tipo na região. Teve por finalidade obter informações cujos resultados pudessem subsidiar tanto o desenvolvimento de estudos complementares, como a organização 
de políticas públicas destinadas aos idosos. A pesquisa original pode ser acessada no sítio http://www.fsp.usp.br/sabe.

Dessa forma, e a partir dos dados do Estudo SABE, o objetivo do presente trabalho foi verificar a ocorrência do uso de AASI pelos idosos com deficiência auditiva autorreferida no município de São Paulo, descrever as razões para o não uso do dispositivo e analisar os possíveis fatores associados ao uso da prótese auditiva.

\section{MÉTODOS}

A população estudada foi de idosos residentes no Município de São Paulo, no ano de 2006, que integraram o estudo SABE, realizado no Brasil.

A amostra foi composta por dois segmentos. O primeiro, resultante de sorteio, baseou-se em cadastro permanente de 72 setores censitários, disponível no Departamento de Epidemiologia da Faculdade de Saúde Pública da Universidade de São Paulo (FSP/USP). Esse segmento da amostra foi obtido no cadastro da Pesquisa Nacional por Amostra de Domicílios (PNAD, 1995), composto por 263 setores censitários, sorteados mediante amostragem por conglomerados, sob o critério de probabilidade proporcional ao número de domicílios. O segmento correspondeu à amostra probabilística formada por 1568 entrevistados.

O segundo segmento, composto por 575 residentes nos distritos em que se realizaram as entrevistas anteriores, correspondeu ao acréscimo realizado para completar o número estabelecido de entrevistas com pessoas com mais de 75 anos, considerando-se o índice de mortalidade na população acima dessa faixa etária. $\mathrm{O}$ número mínimo de domicílios sorteados no segundo segmento foi aproximado para 90. A complementação da amostra de pessoas com idade igual ou superior a 75 anos foi realizada por meio da localização de moradias próximas aos setores selecionados ou, no máximo, dentro dos limites dos distritos aos quais pertenciam os setores sorteados, segundo o estudo SABE. Os dados foram colhidos simultaneamente, por meio de entrevistas domiciliares, feitas com um instrumento constituído por 11 blocos temáticos. Cada questionário teve um peso, calculado de acordo com o setor censitário correspondente (peso=1/f).

Para os questionários realizados com indivíduos em domicílios não sorteados (faixa etária igual ou superior a 75 anos), o cálculo do peso foi efetuado de acordo com a relação da população de idosos nessa faixa etária, residente no Município de São Paulo em 1998, e o número total de idosos dessa faixa encontrado na amostra final do estudo.

Para o presente recorte, a amostra foi composta por 1115 idosos com idade igual ou superior a 65 anos, residentes no município de São Paulo, entrevistados durante o levantamento do Estudo SABE no ano de 2006 e que também haviam sido entrevistados previamente na primeira fase, em 2000. Foram considerados sujeitos com DA autorreferida todos os que preencheram os critérios a seguir, simultânea ou isoladamente: declararam sua audição como regular, ruim ou muito ruim para a questão "Em geral, como o(a) Sr(a) diria que é a sua audição (com ou sem aparelho de audição)"; referiram ser usuários de aparelho auditivo e/ou tiveram indicação prévia de AASI.

Os dados sobre o uso de AASI e a reabilitação foram aferidos pelas questões contidas no bloco C, "Estado de Saúde", a saber: $O(a) \operatorname{Sr}(a)$ usa algum tipo de aparelho auditivo? $O(a)$ $\operatorname{Sr}($ a) não usa aparelho por que? Como o (a) Sr(a) adquiriu o aparelho? $\mathrm{O}($ a) $\mathrm{Sr}($ a) recebeu algum treinamento especial para usar o aparelho? $\mathrm{O}$ (a) $\mathrm{Sr}$ (a) recebeu algum seguimento para controle do funcionamento do aparelho?

Como fatores que poderiam associar-se ao uso de AASI entre os idosos do município de São Paulo, estudou-se as seguintes variáveis que, eventualmente, influenciariam em sua adaptação:

- Dados sociodemográficos: faixa etária, gênero, etnia, organização de domicílio, alfabetização, escolaridade (em anos de estudo), renda (em salários mínimos), percepção de renda suficiente e posição na ocupação.

- Dados de morbidade, locomoção e assistência à saúde: autopercepção da saúde, locomoção, presença de sintomas depressivos, ser considerado caso de provável demência, relatar tontura/vertigem, saber que tem direito ao SUS, possuir plano privado de saúde com cobertura de serviços de reabilitação e adaptação de órteses, próteses ou aparelho de apoio.

- Desempenho em atividades básicas e instrumentais da vida diária.

Definiu-se como casos de provável demência aqueles que obtiveram resultados alterados, simultaneamente, nos testes MEEM (Mini Exame do Estado Mental) e QPAF (Pfeffer Functional Activities Questionnarie - Questionário de Pfeffer para atividades funcionais). O MEEM, inicialmente desenvolvido por Folstein et al. ${ }^{(9)}$, é um instrumento de rastreamento de comprometimento cognitivo, utilizado internacionalmente com o objetivo de fornecer informações sobre diferentes dimensões das funções cognitivas, tais como orientação, memória, cálculo e linguagem. Para o presente estudo, foi aplicada uma versão modificada com ponto de corte de $12 / 13$, sendo o prejuízo cognitivo indicado pela pontuação igual ou inferior a 12.

$\mathrm{O}$ resultado do MEEM foi utilizado como filtro para a aplicação do questionário $\mathrm{QPAF}^{(10)}$, que se destina a avaliar a autonomia funcional da pessoa em relação às atividades da vida cotidiana. O objetivo de se associar o resultado do QPAF foi verificar se à deficiência cognitiva somavam-se limitações da capacidade funcional. O ponto de corte foi o proposto pelos seus autores (igual ou superior a seis, para indicar que o indivíduo necessitava de ajuda para desenvolver atividades cotidianas denominadas instrumentais). Pessoas que obtiveram pontuação 12 ou inferior no MEEM e seis ou superior na QPAF puderam contar com um informante substituto para a realização da entrevista. Aqueles que obtiveram 13 pontos ou mais no MEEM e cinco pontos ou menos na QPAF puderam ter um informante auxiliar para o restante do questionário. 
A análise dos dados foi conduzida utilizando-se o programa Stata $10.0^{\circledR}$, módulo "survey", que permite incorporar aspectos referentes ao delineamento complexo de amostragem, a saber: estratificação não proporcional, sorteio de conglomerados e ponderação. A variável peso, criada para ponderar os dados, foi definida pelo inverso da fração amostral e ajustada para que a amostra não apresentasse distorções no que se refere à idade e ao gênero. A sumarização dos dados foi realizada a partir de análise exploratória, utilizando-se porcentagens para descrever as variáveis categóricas e médias e desvios-padrão para descrição das variáveis contínuas.

Para a análise univariada, aplicou-se o teste de Rao-Scott, que realiza a prova de significância da associação em tabelas de dupla entrada, ajustando-se pelo desenho amostral. A análise multivariada foi realizada por meio da regressão logística, tipo "stepwise backward" (passo a passo para trás). Foram selecionadas para integrar o modelo de regressão logística todas as variáveis com associação com à variável dependente, com nível de significância de $20 \%$ ( $<<0,20$ ). Como segunda possibilidade, integraram a análise múltipla as variáveis que a literatura enfatiza como fortemente associadas ao desfecho estudado e/ou que pudessem ser fatores de confusão com outras variáveis, mesmo que a força de associação não tivesse sido verificada no modelo univariado (tal como gênero e idade). Utilizou-se o procedimento retrógrado (ou passo a passo para trás) para a elaboração do modelo múltiplo e a variável permaneceu no modelo final de $p<0,05$. A força da associação entre as variáveis independentes e a variável dependente foi expressa em valores estimados, brutos e ajustados, de razões de prevalência com intervalos de confiança de $95 \%$ (IC 95\%).

O projeto de pesquisa do Estudo SABE foi analisado e aprovado pelo Comitê de Ética em Pesquisa da Faculdade de Saúde Pública da Universidade de São Paulo, protocolo número 1345 , ofício COEP/83/06, tendo sido obtida a autorização para a análise dos dados. Todos os sujeitos avaliados assinaram o Termo de Consentimento Livre e Esclarecido.

\section{RESULTADOS}

Foram entrevistados 1115 idosos, com média de idade de 68,1 anos $(\mathrm{DP} \pm 0,4)$. A prevalência de $\mathrm{DA}$ autorreferida foi de 30,4\% (IC95\% 27,2 a 33,8), representada por 377 idosos.

A prevalência de usuários de AASI na população total estudada ( $\mathrm{n}=1115$ ) foi de 3\% (IC95\% 1,9 a 4,7\%). Entretanto, se considerarmos apenas os idosos com DA autorreferida $(n=377)$, a prevalência de usuários do dispositivo foi de 10,1\% (IC95\% 6,5 a 15,2). O estudo do processo da concessão do dispositivo eletrônico e posterior acompanhamento do idoso usuário de AASI constam na Tabela 1.

As respostas obtidas pelos entrevistadores, durante a aplicação do instrumento, com a indicação das opções "não sabe/ não respondeu", entendidas como «não respostas», não foram
Tabela 1. Distribuição de número e porcentagem do uso de AASI por idosos com DA autorreferida

\begin{tabular}{lcc}
\hline Variável & $\mathrm{n}$ & $\%$ \\
\hline Usa AASI & 45 & 10,1 \\
Sim & 332 & 89,9 \\
Não & & \\
Por que não usa & 132 & 43,9 \\
$\quad$ Não precisa & 130 & 39,5 \\
Nunca foi indicado & 33 & 8,6 \\
Foi indicado, mas não se acostumou & 28 & 8,0 \\
Foi indicado, mas não teve dinheiro para comprar & 323 & 100 \\
Total & & \\
Como adquiriu o AASI & 23 & 56,1 \\
Próprio idoso pagou & 11 & 22,7 \\
Outra pessoa pagou & 06 & 16,9 \\
SUS & 01 & 1,8 \\
Convênio/plano de saúde & 03 & 2,5 \\
Outros & 44 & 100 \\
Total & & \\
Recebeu treinamento para o uso do AASI & 34 & 87,0 \\
Sim & 10 & 13,0 \\
Não & 44 & 100 \\
Total & & \\
Recebeu seguimento para controle do AASI & 33 & 81,4 \\
Sim & 10 & 18,6 \\
Não & 43 & 100 \\
Total & & \\
\hline
\end{tabular}

Legenda: $\mathrm{AASI}=$ Aparelho de Amplificação Sonora Individual

contabilizadas nas tabelas finais, sendo, dessa forma, consideradas perdas amostrais do presente estudo. A decisão de não considerá-las para o processamento deveu-se à sua possível influência nos valores exatos de prevalências totais obtidas, ou distribuições descritas, de acordo com a metodologia estatística escolhida para análise.

A necessidade de um informante auxiliar (proxy) para o levantamento de dados foi maior entre os idosos não usuários de AASI, comparando-se aos idosos que fizeram uso do dispositivo $(13,8 \%$ versus $1,7 \% \mathrm{p}<0,001)$. A análise univariada dos fatores associados ao uso do AASI consta das Tabelas 2, 3, 4.

Todas as associações estabelecidas na análise univariada com um valor de $\mathrm{p}<0,20$ foram incluídas na regressão logística. Segundo esse critério, foram incluídas na análise multivariada as variáveis independentes, a saber: posição na ocupação, provável demência, possuir plano privado de saúde, dificuldades nas atividades básicas da vida diária (vestir-se, utilizar o banheiro) dificuldades em todas as atividades instrumentais da vida diária, e, especificamente, utilizar o telefone e ingerir medicamentos, sendo o desfecho dessa análise o uso ou não de AASI. O modelo final da regressão logística foi ajustado para o gênero e a faixa etária, devido à possibilidade dessas variáveis apresentarem-se como fator de confusão no modelo estudado. Dessa forma, as variáveis que permaneceram no modelo, com $\mathrm{p}<0,05$ após a análise multivariada, estão descritas na Tabela 5. 
Tabela 2. Distribuição de número e porcentagem do uso de AASI por idosos com DA autorreferida segundo fatores sociodemográficos

\begin{tabular}{|c|c|c|c|c|c|}
\hline \multirow{3}{*}{ Variável } & \multicolumn{4}{|c|}{ Usa AASI } & \multirow{3}{*}{ Valor de } \\
\hline & \multicolumn{2}{|c|}{ Sim } & \multicolumn{2}{|c|}{ Não } & \\
\hline & $\mathrm{N}$ & $\%$ & $\mathrm{n}$ & $\%$ & \\
\hline \multicolumn{6}{|l|}{ Faixa etária } \\
\hline $65-69$ & 11 & 52,9 & 110 & 53,0 & \multirow{5}{*}{0,24} \\
\hline $70-74$ & 6 & 26,5 & 73 & 15,9 & \\
\hline $75-79$ & 11 & 12,7 & 81 & 15,4 & \\
\hline $80-84$ & 13 & 4,6 & 40 & 12,7 & \\
\hline 85 e mais & 4 & 3,3 & 26 & 3,0 & \\
\hline \multicolumn{6}{|l|}{ Gênero } \\
\hline Feminino & 24 & 59,6 & 181 & 53,1 & \multirow{2}{*}{0,43} \\
\hline Masculino & 21 & 40,4 & 151 & 46,9 & \\
\hline \multicolumn{6}{|l|}{ Cor/etnia } \\
\hline Branco & 27 & 67,9 & 231 & 68,3 & \multirow{2}{*}{0,97} \\
\hline Outras & 18 & 32,1 & 100 & 31,7 & \\
\hline \multicolumn{6}{|l|}{ Organização do domicílio } \\
\hline Mora sozinho & 8 & 9,5 & 51 & 14,9 & \multirow{2}{*}{0,39} \\
\hline Mora acompanhado & 36 & 90,5 & 279 & 85,1 & \\
\hline \multicolumn{6}{|l|}{ Alfabetizado } \\
\hline Sim & 39 & 81,6 & 259 & 80,6 & \multirow{2}{*}{0,91} \\
\hline Não & 6 & 18,4 & 73 & 19,4 & \\
\hline \multicolumn{6}{|l|}{ Escolaridade (anos de estudo) } \\
\hline Nenhum & 9 & 20,6 & 100 & 25,1 & \multirow{3}{*}{0,46} \\
\hline 1 a 6 & 25 & 55,5 & 194 & 59,8 & \\
\hline 7 a 11 & 11 & 23,9 & 38 & 15,9 & \\
\hline \multicolumn{6}{|l|}{ Renda (salários mínimos) } \\
\hline Menos que 01 & 2 & 1,8 & 09 & 4,5 & \multirow{2}{*}{0,23} \\
\hline Igual ou superior a 01 & 28 & 98,2 & 220 & 95,5 & \\
\hline \multicolumn{6}{|l|}{ Percepção de renda } \\
\hline Suficiente & 28 & 44,4 & 155 & 46,9 & \multirow{2}{*}{0,89} \\
\hline Insuficiente & 17 & 55,6 & 164 & 53,1 & \\
\hline \multicolumn{6}{|l|}{ Posição na ocupação } \\
\hline Operário/trabalhador/rural & 29 & 80,6 & 186 & 59,9 & \multirow{4}{*}{$0,001^{*}$} \\
\hline Autônomo & 02 & 2,4 & 12 & 3,5 & \\
\hline Patrão & 05 & 4,9 & 84 & 30,4 & \\
\hline Outros & 06 & 12,1 & 23 & 6,2 & \\
\hline
\end{tabular}

${ }^{*}$ Valores significativos $(p \leq 0,05)$ - Teste de Rao-Scott

Legenda: $\mathrm{AASI}=$ Aparelho de Amplificação Sonora Individual

\section{DISCUSSÃO}

Verificou-se baixo índice de uso de AASI $(10,1 \%)$ entre os idosos com DA autorreferida no município de São Paulo, porcentagem inferior à relatada em estudo realizado na Finlândia, que foi de 15 a $30 \%{ }^{(7)}$. Além dos valores mais baixos, os motivos para o não uso também foram distintos. No presente estudo, uma das principais razões atribuídas para o não uso do aparelho foi o fato de o idoso não considerá-lo necessário, ou então, por nunca ter recebido indicação para seu uso. Segundo dados revelados no Japão ${ }^{(11)}$, que avaliaram população de 40 a 84 anos, constatou-se que $11,0 \%$ dos sujeitos com DA eram usuários de AASI, valor bem próximo ao detectado no presente estudo, apesar da diferença entre as faixas etárias consideradas. No referido estudo, os autores salientaram que o uso do dispositivo costumava diminuir com o avanço da idade, sendo também influenciado por outras variáveis, como gênero e nível educacional.

Embora a DA seja muito comum na população idosa, as opções de tratamento podem não ser bem aceitas, principalmente quando a deficiência não é profunda ${ }^{(12)}$. Nos EUA, apenas $25 \%$ dos adultos com DA fazem uso de AASI e os motivos descritos foram semelhantes aos do presente estudo, como por exemplo, a não percepção da necessidade do aparelho ${ }^{(13)}$. Uma explicação para esse fato seria que a procura pelo AASI geralmente ocorre de dez a 13 anos após a primeira detecção da DA, quando esta já se encontra em estágios avançados, ou tornou-se uma DA profunda ${ }^{(14)}$.

No Brasil, a demora em procurar o atendimento profissional para a DA indicou que a ajuda especializada para o atendimento auditivo é o último recurso a ser procurado pelos idosos ${ }^{(15)}$. Mesmo assim, após a decisão de buscar ajuda profissional, os 
Tabela 3. Distribuição de número e porcentagem do uso de AASI por idosos com DA autorreferida segundo morbidades, locomoção e assistência à saúde

\begin{tabular}{|c|c|c|c|c|c|}
\hline \multirow{3}{*}{ Variável } & \multicolumn{4}{|c|}{ Usa AASI } & \multirow{3}{*}{ Valor de $p$} \\
\hline & \multicolumn{2}{|c|}{ Sim } & \multicolumn{2}{|c|}{ Não } & \\
\hline & $\mathrm{n}$ & $\%$ & $\mathrm{n}$ & $\%$ & \\
\hline \multicolumn{6}{|l|}{ Autopercepção de saúde } \\
\hline Muito boa ou boa & 22 & 49,6 & 119 & 39,2 & \multirow{2}{*}{0,29} \\
\hline Regular, Ruim ou Muito Ruim & 23 & 50,4 & 210 & 60,8 & \\
\hline \multicolumn{6}{|l|}{ Locomoção } \\
\hline Deambulante & 45 & 100 & 317 & 97,2 & \multirow{2}{*}{0,23} \\
\hline Cadeirante/acamado & - & - & 14 & 2,8 & \\
\hline \multicolumn{6}{|l|}{ Sintomas depressivos } \\
\hline Caso & 5 & 10,8 & 56 & 20,2 & \multirow{2}{*}{0,33} \\
\hline Não caso & 38 & 89,2 & 213 & 79,8 & \\
\hline \multicolumn{6}{|l|}{ Provável demência } \\
\hline Caso & 2 & 1,8 & 70 & 14,7 & \multirow{2}{*}{$0,002^{*}$} \\
\hline Não caso & 42 & 98,2 & 241 & 85,3 & \\
\hline \multicolumn{6}{|l|}{ Tontura/vertigem } \\
\hline Sim & 5 & 18,1 & 75 & 26,5 & \multirow[t]{2}{*}{0,34} \\
\hline Não & 38 & 81,9 & 211 & 73,5 & \\
\hline \multicolumn{6}{|l|}{ Sabe que tem direito ao SUS } \\
\hline Sim & 43 & 93,9 & 314 & 96,9 & \multirow{2}{*}{0,41} \\
\hline Não & 2 & 6,1 & 11 & 3,1 & \\
\hline \multicolumn{6}{|l|}{ Plano privado de saúde } \\
\hline Sim & 30 & 60,6 & 145 & 43,8 & \multirow{2}{*}{0,05} \\
\hline Não & 15 & 39,4 & 186 & 56,2 & \\
\hline \multicolumn{6}{|c|}{ Plano privado cobre serviços de reabilitação } \\
\hline Sim & 17 & 74,9 & 91 & 80,8 & \multirow{2}{*}{0,61} \\
\hline Não & 05 & 25,1 & 22 & 19,2 & \\
\hline \multicolumn{6}{|c|}{ Plano privado cobre próteses, órteses ou aparelhos de apoio } \\
\hline Sim & 3 & 14,0 & 13 & 12,8 & \multirow{2}{*}{0,90} \\
\hline Não & 20 & 86,0 & 89 & 87,2 & \\
\hline
\end{tabular}

*Valores significativos $(p \leq 0,05)-$ Teste de Rao-Scott

Legenda: AASI = Aparelho de Amplificação Sonora Individual

idosos depararam-se com barreiras para o acesso aos especialistas e para a adesão ao tratamento, visto não encontrarem a "cura" para a DA e terem suas expectativas frustradas.

Frente aos resultados do presente estudo, pôde-se levantar duas possíveis explicações para esses casos: a procura por um profissional para o tratamento da DA ainda é baixa, possivelmente pela dificuldade em encontrar serviços disponíveis na comunidade, ou pela dificuldade desses serviços atenderem às expectativas dos idosos; o início lento e gradual da perda auditiva, característica da presbiacusia, que poderia favorecer o avanço da DA, sem que medidas fossem tomadas precocemente. Além disso, o estigma pelo uso do AASI poderia gerar recusa em aceitação, o que justificaria a resposta "não achava necessário".

Em estudo que explorou o discurso de cinco idosos usuários de AASI, para a identificação de repertórios que justificassem a decisão pelo uso do dispositivo, autores brasileiros encontraram as seguintes justificativas: o incômodo da repetição, a busca da prevenção e autocuidado, o aproveitamento de um recurso tecnológico benéfico, os benefícios da prótese, superando as limitações, e a virtude da resignação, já que, como tinham a DA e a aceitavam, poderiam não ser atingidos por outros "malefícios"(16).

Outra vertente explicativa para a decisão sobre o uso do AASI, remete ao conceito de satisfação. Apesar do avanço tecnológico dos sistemas acústicos modernos, a satisfação do usuário continua sendo um desafio para os audiologistas e as altas taxas de abandono do uso do AASI, um problema para os serviços de saúde ${ }^{(17)}$. Sendo assim, o monitoramento da satisfação é importante para avaliar os procedimentos clínicos e garantir os propósitos de qualidade dos serviços de saúde. Estudos indicam que, usuários de AASI atendidos pelo SUS e avaliados por meio do questionário SADL (Satisfaction with Amplification in Daily Life) adaptado para o português, encontravam-se satisfeitos com a adaptação, sendo a escala imagem pessoal a mais beneficiada ${ }^{(18)}$. Em outra abordagem com o mesmo instrumento, concluiu-se que o grau de satisfação de adultos com os dispositivos fornecidos pelo sistema público de saúde teve relação com o tipo de AASI, sendo o intra-aural o melhor avaliado. $\mathrm{O}$ grau de satisfação não teve relação com idade, gênero, grau da perda auditiva e perfil eletrofisiológico ${ }^{(19)}$. 
Tabela 4. Distribuição de número e porcentagem do uso de AASI por idosos com DA autorreferida segundo desempenho em atividades básicas e instrumentais de vida diária

\begin{tabular}{|c|c|c|c|c|c|}
\hline Variável & $\mathrm{n}$ & Com AASI (\%) & $\mathrm{n}$ & Sem AASI (\%) & Valor de $p$ \\
\hline \multicolumn{6}{|l|}{ Dificuldade em ABVD* } \\
\hline Uma ou mais dificuldades & 32 & 81,1 & 207 & 70,9 & \multirow{2}{*}{0,22} \\
\hline Sem dificuldades & 13 & 18,9 & 122 & 29,1 & \\
\hline \multicolumn{6}{|l|}{ ABVD } \\
\hline \multicolumn{6}{|l|}{ Caminhar } \\
\hline Com dificuldade & 4 & 5,6 & 56 & 11,1 & \multirow{2}{*}{0,29} \\
\hline Sem dificuldade & 41 & 94,4 & 275 & 88,9 & \\
\hline \multicolumn{6}{|l|}{ Vestir-se } \\
\hline Com dificuldade & 9 & 12,0 & 60 & 23,8 & \multirow{2}{*}{0,15} \\
\hline Sem dificuldade & 36 & 88,0 & 271 & 76,2 & \\
\hline \multicolumn{6}{|l|}{ Tomar banho } \\
\hline Com dificuldade & 8 & 7,8 & 60 & 11,6 & \multirow{2}{*}{0,40} \\
\hline Sem dificuldade & 37 & 92,2 & 271 & 88,4 & \\
\hline \multicolumn{6}{|l|}{ Comer sozinho } \\
\hline Com dificuldade & 3 & 3,3 & 25 & 4,9 & \multirow{2}{*}{0,66} \\
\hline Sem dificuldade & 42 & 96,7 & 304 & 95,1 & \\
\hline \multicolumn{6}{|l|}{ Levantar da cama } \\
\hline Com dificuldade & 8 & 10,7 & 74 & 17,2 & \multirow{2}{*}{0,31} \\
\hline Sem dificuldade & 37 & 89,3 & 256 & 82,8 & \\
\hline \multicolumn{6}{|l|}{ Utilizar banheiro } \\
\hline Com dificuldade & 4 & 3,0 & 49 & 9,3 & \multirow{2}{*}{0,07} \\
\hline Sem dificuldade & 41 & 97,0 & 282 & 90,7 & \\
\hline \multicolumn{6}{|l|}{ Dificuldade em AIVD ${ }^{\star *}$} \\
\hline Uma ou mais dificuldades & 05 & 8,5 & 52 & 21,8 & \multirow{2}{*}{0,13} \\
\hline Sem dificuldades & 40 & 91,5 & 273 & 78,2 & \\
\hline \multicolumn{6}{|l|}{ AIVD } \\
\hline \multicolumn{6}{|l|}{ Preparar refeição quente } \\
\hline Com dificuldade & 18 & 37,2 & 155 & 39,7 & \multirow{2}{*}{0,75} \\
\hline Sem dificuldade & 26 & 62,8 & 176 & 60,3 & \\
\hline \multicolumn{6}{|l|}{ Cuidar do dinheiro } \\
\hline Com dificuldade & 11 & 19,2 & 113 & 25,2 & 807 \\
\hline Sem dificuldade & 33 & 80,8 & 216 & 74,8 & $0,4 \pi$ \\
\hline Utilizar transporte público & & & & & \\
\hline Com dificuldade & 22 & 45,2 & 168 & 38,6 & 042 \\
\hline Sem dificuldade & 23 & 54,8 & 162 & 61,4 & 0,42 \\
\hline Comprar alimentos & & & & & \\
\hline Com dificuldade & 18 & 27,7 & 148 & 41,1 & ?20 \\
\hline Sem dificuldade & 27 & 72,3 & 183 & 58,9 & $0, \angle 2$ \\
\hline Usar o telefone & & & & & \\
\hline Com dificuldade & 09 & 14,2 & 110 & 25,8 & 017 \\
\hline Sem dificuldade & 36 & 85,8 & 219 & 74,2 & 0,17 \\
\hline Tarefas domésticas leves & & & & & \\
\hline Com dificuldade & 23 & 50,6 & 169 & 45,3 & \\
\hline Sem dificuldade & 22 & 49,4 & 161 & 54,7 & $0,0 r$ \\
\hline Tarefas domésticas pesadas & & & & & \\
\hline Com dificuldade & 37 & 77,6 & 244 & 68,2 & קח? \\
\hline Sem dificuldade & 08 & 22,4 & 87 & 31,8 & 0,32 \\
\hline Tomar remédios & & & & & \\
\hline Com dificuldade & 07 & 8,1 & 107 & 24,9 & $0 \Omega 1 *$ \\
\hline Sem dificuldade & 38 & 91,9 & 224 & 75,1 & $0,07^{n}$ \\
\hline
\end{tabular}

*Valores significativos $(p \leq 0,05)$ - Teste de Rao-Scott

Legenda: $\mathrm{AASI}=$ Aparelho de Amplificação Sonora Individual; $\mathrm{ABVD}=$ atividades básicas de vida diária; $\mathrm{AIVD}=$ atividades instrumentais de vida diária 
Tabela 5. Regressão Logística para o uso de AASI em idosos com DA autorreferida

\begin{tabular}{|c|c|c|c|c|c|c|}
\hline \multirow{3}{*}{ Variável } & \multicolumn{4}{|c|}{ USA AASI } & \multirow{3}{*}{$\begin{array}{l}\text { OR ajustada } \\
\text { (IC 95\%) }\end{array}$} & \multirow{3}{*}{ Valor de $\mathrm{p}$} \\
\hline & \multicolumn{2}{|c|}{ Sim } & \multicolumn{2}{|c|}{ Não } & & \\
\hline & $\mathrm{n}$ & $\%$ & $\mathrm{n}$ & $\%$ & & \\
\hline \multicolumn{7}{|l|}{ Provável demência } \\
\hline Caso & 02 & 1,8 & 70 & 14,7 & $11,3(3,8-33,7)$ & \multirow{2}{*}{$<0,001^{*}$} \\
\hline Não Caso & 42 & 98,2 & 241 & 85,3 & 1,0 & \\
\hline \multicolumn{7}{|l|}{ Utilizar banheiro } \\
\hline Com dificuldade & 04 & 3,0 & 49 & 9,3 & $5,7(1,8-18,2)$ & \multirow{2}{*}{$0,003^{*}$} \\
\hline Sem dificuldade & 41 & 97,0 & 282 & 90,7 & 1,0 & \\
\hline
\end{tabular}

* Valores significativos $(p \leq 0,05)$ e ajustados por gênero e faixa etária (Odds Ratio - OR), Regressão Logística

Legenda: $\mathrm{AASI}=$ Aparelho de Amplificação Sonora Individual

No presente estudo, destacou-se a forma de obtenção do dispositivo pelos usuários de AASI, tendo a maioria adquirido o aparelho com recursos privados. Observou-se, com relação especificamente à aquisição e uso do AASI, que a adesão ao SUS (Sistema Único de Saúde) pelos idosos, para o tratamento de sua DA, ainda é baixa, fato importante, tendo em vista o alto custo do dispositivo.

A Política Nacional de Atenção à Saúde Auditiva (PNSA) tem por objetivo nortear as ações de saúde auditiva e definir os papéis dos serviços, a fim de ampliar o acesso e organizar os atendimentos ${ }^{(3)}$. Considerando que a coleta dos dados desta pesquisa ocorreu apenas dois anos após a instituição da PNSA (2006), espera-se que o fornecimento do AASI pelo SUS tenha se intensificado após a efetiva implantação e/ou maior divulgação da Política.

Esta última colocação é fortalecida pela literatura ${ }^{(6,20)}$. Em uma coleta realizada em Pernambuco, no ano de 2003, observou-se que $63 \%$ dos municípios não possuíam nenhum profissional da área de diagnóstico da deficiência auditiva, fossem médicos otorrinolaringologistas, ou fonoaudiólogos, cadastrados no Sistema de Informação Ambulatorial do Ministério da Saúde ${ }^{(20)}$. Acredita-se, portanto, que nesses municípios, não se oferecia à população nenhuma ação que promovesse a saúde auditiva, sequer na atenção básica.

Posteriormente, em 2006, um estudo qualitativo sobre a percepção dos idosos sobre o uso de AASI concedido pelo SUS, no Estado de Santa Catarina, revelou que o que possibilitou o acesso ao AASI foi a implantação do Serviço de Atenção à Saúde Auditiva na região ${ }^{(6)}$. Entretanto, verificou-se a crença dos idosos de que nos serviços privados de saúde teriam a possibilidade de receber um AASI de melhor qualidade do que o concedido por meio do SUS, apesar da grande oferta de aparelhos disponibilizados pelo sistema público.

No presente estudo, a maioria dos idosos usuários de AASI $(87 \%)$ declarou ter recebido treinamento para a utilização do dispositivo e acompanhamento para orientação do seu uso $(81,4 \%)$. Nessa perspectiva, ressalta-se que o processo de adaptação ao uso do AASI é especialmente difícil para os idosos porque, geralmente, requerem mais tempo para assimilar todas as etapas do processo de seleção e adaptação à amplificação, e, em consequência, é necessário que as informações sejam apresentadas de forma gradual e contínua ${ }^{(21)}$. Entretanto, mesmo com possíveis dificuldades, verificou-se que os idosos que declararam ser usuários de AASI relataram ter recebido orientação e acompanhamento, fator que provavelmente interferiu positivamente no uso do dispositivo.

Outra análise realizada pelo presente estudo focalizou os fatores associados ao uso do AASI entre idosos no município de São Paulo e, dentre esses fatores, o contato com os serviços de saúde. A grande maioria dos idosos usuários de AASI (93,9\%) relatou saber que tem direito ao SUS, mas essa porcentagem também foi elevada entre os não usuários $(96,6 \%)$. Porém, há que se considerar como uma limitação para a análise desse resultado a forma genérica da abordagem dessa questão, ou seja, não se questionou diretamente se o idoso sabia do seu direito ao SUS especificamente para o atendimento audiológico.

Por meio da análise multivariada, constatou-se que somente a presença de provável demência e a dificuldade no desempenho da atividade de vida diária de utilização do banheiro, associaram-se ao uso de AASI. Frente a esses achados, as seguintes hipóteses foram levantadas: o uso do aparelho auditivo poderia ser um fator de proteção para a não ocorrência de demência, visto que a amplificação sonora proporcionada pelo uso do dispositivo poderia favorecer a preservação de algumas habilidades cognitivas; idosos com processos demenciais instalados poderiam apresentar maiores dificuldades nos exames auditivos, sobretudo os comportamentais (audiometria e logoaudiometria), e no cumprimento dos protocolos de adaptação de AASI, fatores que prejudicariam o processo de diagnóstico auditivo e adaptação nessa população, principalmente se metodologias objetivas não estivessem disponíveis. Entretanto, pelo desenho transversal do presente estudo, não foi possível afirmar qual dos fatores ocorreu primeiro. Dessa forma, a descrição exata da causalidade nas situações mencionadas não pode ser considerada viável, configurando-se como uma limitação do estudo.

A hipótese de que o uso do AASI poderia ser um protetor para a manutenção do desempenho cognitivo de idosos encontra respaldo na literatura nacional e internacional. Estudos demonstraram que a reabilitação auditiva com AASI propiciou melhora em medidas cognitivas globais ${ }^{(22)}$, ocorrência percebida por familiares próximos. A habilidade mais favorecida pelo uso da prótese seria a atenção auditiva, o que contribuiria para a 
diminuição do isolamento social e da dificuldade comunicativa, e, portanto, para a melhoria na qualidade de $\operatorname{vida}^{(23)}$.

Outra hipótese levantada foi a de que idosos com deficiência auditiva teriam mais dificuldade no desempenho do teste MEEM e poderiam receber pior avaliação de seus cuidadores no QPAF, visto que a execução de todos os itens do MEEM depende da compreensão de comandos orais e muitas das atividades avaliadas no QFAF dependem da audição. Este viés poderia produzir um número expressivo de casos falsos de "provável demência", uma vez que a dificuldade auditiva, sem a compensação da prótese, poderia ter influenciado a avaliação cognitiva e funcional desses idosos.

A relação entre comprometimento cognitivo e DA vem sendo assinalada em estudos relacionados ao envelhecimento $^{(24)}$. Estudo específico ${ }^{(25)}$ relatou um risco 6,6 vezes maior de pacientes com declínio cognitivo apresentarem DA, em relação ao grupo controle. Nessa temática, autores estadunidenses estudaram o tempo de reação motora após um estímulo auditivo e encontraram alterações na modulação do córtex auditivo em sujeitos com declínio cognitivo, resultado de alterações neurológicas em áreas do córtex associativo ${ }^{(26)}$. Além da explicação biológica, o fator ambiental também poderia contribuir para a presença do declínio em deficientes auditivos. A DA pode acarretar isolamento social, depressão e redução da capacidade funcional ${ }^{(27)}$, fatores que podem produzir um falso diagnóstico de demência. Desse modo, os audiologistas precisam reconhecer a importância das funções cognitivas nas habilidades auditivas, pois o ouvir, no cotidiano, é altamente influenciado pela cognição. Por outro lado, deficiências sensoriais, tais como as auditivas e motoras, podem amplificar ou mascarar declínios cognitivos ${ }^{(28)}$. Apesar dessa relação ainda ser pouco enfatizada na formação de fonoaudiólogos e médicos otorrinolaringologistas, a diferenciação diagnóstica entre declínio cognitivo e DA deve ser cuidadosamente observada para que o diagnóstico e a intervenção sejam corretos, principalmente na população idosa, que apresenta prevalências maiores de ambas as morbidades ${ }^{(29)}$.

O uso do AASI pode se relacionar com o desempenho das pessoas em várias atividades da vida diária, principalmente as instrumentais que envolvem o uso de comunicação para seu desempenho. Entretanto, neste estudo, apenas associou-se ao uso de AASI a atividade de utilizar o banheiro, atividade básica da vida diária e que não envolve demandas comunicativas diretas. Uma explicação para essa associação seria que, como o não uso de AASI relacionou-se à maior prevalência de provável demência, idosos não usuários de AASI seriam os mais comprometidos cognitivamente e necessitariam de ajuda para as necessidades cotidianas mais básicas. Outro fator considerado remeteu ao efeito de proxy. Como muitos idosos não usuários de AASI necessitaram do informante substituto para responder ao questionário e a atividade de auxílio para a utilização do banheiro costuma ser uma das mais difíceis de serem desempenhadas pelo cuidador, essa informação poderia ter se sobressaído, demonstrando um relato mais acentuado desse cuidador frente uma dificuldade cotidiana enfrentada por ambos (idoso e cuidador).

Um importante dado observado foi o número de informantes auxiliares que os idosos com DA autorreferida, não usuários de AASI, necessitaram para responder ao questionário SABE. Esse fato pode ter gerado algumas respostas imprecisas (efeito de proxy) e colaborado para o número de "não respostas" em determinadas tabelas, configurando-se uma limitação do presente estudo. Outra provável explicação para esses missings pode ser encontrada nas dificuldades comunicativas que a DA poderia provocar. Muitas das questões podem ter sido assinaladas como "não sabe/não respondeu" pelo não entendimento da pergunta pelo idoso, como decorrência da dificuldade de detecção sonora ou de processamento da informação acústica. Como segunda hipótese, pode ter ocorrido dificuldade de entendimento do entrevistador à resposta, visto que o prejuízo no feedback auditivo do idoso pode acarretar problemas de fala, bem como distúrbios vocais ${ }^{(30)}$, situações que levariam à diminuição do total de respostas consideradas, em algumas questões. Apesar dessa dificuldade para o estudo dos fatores associados à DA e ao uso do AASI, principalmente os referidos, o seu detalhamento é de suma importância para o conhecimento dessa população e para direcionar ações de saúde coletiva.

\section{CONCLUSÃO}

A porcentagem de idosos com DA autorreferida, usuários de AASI no município de São Paulo, foi considerada baixa, bem como insuficiente a cobertura do SUS para a adaptação e acompanhamento desses usuários. Um dos problemas que o não uso de AASI pode acarretar, ou agravar, é a instalação ou acentuação do declínio cognitivo e a consequente diminuição da capacidade funcional nessa população, visto que o não uso do AASI associou-se à maior prevalência de provável demência, conforme dados revelados pelo presente estudo.

\section{REFERÊNCIAS}

1. Gates GA, Mills JH. Presbycusis. Lancet. 2005Sep;366(9491):111120.

2. Koopmann Jr CF. Problemas otolaringológicos no idoso. Med Clin North Am. 1991;6:1411-27.

3. Brasil. Ministério da Saúde. Politica Nacional de Atenção à Saúde Auditiva. Ato Portaria No 2.073/GM de 28 de setembro de 2004. [Internet] [citado 2010 jun]. Disponível em: http://www.fonosp.org. br/publicar/conteudo2.php?id=298.

4. Travassos C, Martins M. Uma revisão sobre os conceitos de acesso e utilização de serviços de saúde. Cad Saúde Pública. 2004, 20:190-8.

5. Brasil. Ministério da Saúde. Ato Portaria No 154 de 24 de janeiro de 2008. [Internet] [citado $2010 \mathrm{dez}$ ]. Disponível em: http://www.cref6. org.br/arquivos/PORTARIA_n154.pdf. 
6. Fialho IM, Bortoli D, Mendonça GG, Pagnosim DF, Scholze AS. Percepção de idosos sobre o uso de AASI concedido pelo Sistema Único de Saúde. Rev CEFAC. 2009;11(2):338-44.

7. Lupsakko TA, Kautiainen HJ, Sulkava R. The non use of hearing aids in people aged 75 years and over in the city of Kuopio in Finland. Eur Arch Otorhinolaryngol. 2005;262(3):165-9.

8. Gatehouse S, Naylor G, Elberling C. Linear and non-linear hearing aid fittings--2. Patterns of candidature. Int J Audiol. 2006Mar;45(3):153-71.

9. Folstein MF, Folstein SE, Mchugh PR. Mini-mental state. A practical method for grading the cognitive state of patient for the clinician. J Psychiatr Res. 1975Nov;12:189-98.

10. Pfeffer RI, Kurosaki TT, Harrah CH Jr, Chance JM, Filos S. Measurement of functional activities in older adults in the community. J Gerontol. 1987May;37(3):323-9.

11. Uchida Y, Sugiura S, Ando F, Shimokata H, Yoshioka M, Nakashima T. Analyses of factors contributing to hearing aids use and both subjective and objective estimates of hearing. Nippon Jibiinkoka Gakkai Kaiho. 2008May;111(5):405-11.

12. Pratt SR. Barriers to hearing health care: current status and a glimpse at the future. Am J Audiol. 2010Jun;19(1):1-2.

13. Kochkin S. MarkeTrack VII: Obstacles to adult non-user adoption of hearing aids. Hear J. 2007Apr;60(4):24-50.

14. Davis A, Smith P, Ferguson M, Stephens D, Gianopoulos I. Acceptability, benefit, and costs of early screening for hearing disability: a study of potential screening tests and models. Health Technol Assess. 2007Oct;11(42):1-294.

15. Scheffer JC, Fialho IM, Scholze AS. Itinerários de cura e cuidado de idosos com perda auditiva. Saúde Soc. 2009;8(3):537-48.

16. Ribeiro LM, Rasera EF. Os idosos e o uso de próteses auditivas: identificando os repertórios interpretativos que justificam essa decisão. Psicol Soc. 2008;20(3):425-33.

17. Veiga LR, Merlo ARC, Mengue SS. Satisfação com a prótese auditiva na vida diária em usuários do Sistema de Saúde do Exército. Rev Bras Otorrinolaringol. 2005;71(1):67-73.

18. Danieli F, Castiquini EAT, Zambonatto TCF, Bevilacqua MC. Avaliação do nível de satisfação de usuários de aparelhos de amplificação sonora individuais dispensados pelo Sistema Único de Saúde. Rev Soc Bras Fonoaudiol. 2011;16(2):152-9.
19. Farias RB, Russo ICP. Saúde auditiva: estudo do grau de satisfação de usuários de aparelho de amplificação sonora individual. Rev Soc Bras Fonoaudiol. 2010;15(1):26-31.

20. Miranda GMD, Queiroga BAM, Lessa FJD, Leal MC, Caldas Neto SS. Diagnóstico da deficiência auditiva em Pernambuco: oferta de serviços de média complexidade - 2003. Rev Bras Otorrinolaringol. 2006;72(5):581-586.

21. Vieira EP, Miranda EC, Calais LL, Carvalho LMA, Iório MCA, Borges ACLC. Proposta de acompanhamento em grupo para idosos protetizados. Rev Bras Otorrinolaringol. 2007;73(6):752-8.

22. Allen NH, Burns A, Newton V, Hickson F, Ramsden R, Rogers J, et al. The effects of improving hearing in dementia. Age Ageing. 2003;32(2):189-93.

23. Silva AS, Venites JB, Bilton T. A relação entre o uso do aparelho de amplificação sonora individual (AASI) e a melhora da função cognitiva no envelhecimento. Disturb Comun. 2002;14(1):63-89.

24. Silva ML, McLaughlin MT, Rodrigues EJ, Broadbent JC, Gray AR, Hammond-Tooke GD. A Mini-Mental Status Examination for the hearing impaired. Age Ageing. 2008Sep;37(5):593-5.

25. Lopes LC, Magaldi RM, Gândara MER, Reis ACB, Jacob-Filho W. Prevalence of hearing impairment in patients with mild cognitive impairment. Dement Neuropsychol. 2007;3:253-9.

26. Golob EJ, Johnson JK, Starr A. Auditory event-related potentials during target detection are abnormal in mild cognitive impairment. Clin Neurophysiol. 2002Jan;113(1):151-61.

27. Cacciatore F, Napoli C, Abete P, Marciano E, Triassi M, Rengo F. Quality of life determinants and hearing function in an elderly population: Osservatorio Geriatrico Campano Study Group. Gerontology. 1999;45(6):323-8.

28. Pichora-Fuller MK, Singh G. Effects of age on auditory and cognitive processing: implications for hearing aid fitting and audiologic rehabilitation. Trends Amplif. 2006;10:29-59.

29. Zalewski TR. Cognitive decline or hearing loss. Perspect Gerontol. 2010;15:12-18.

30. Prado AC. Principais características da produção vocal do deficiente auditivo. Rev CEFAC. 2007;9(3):404-410. 